\title{
Opposite effects of the gap junction blocker octanol on focal cerebral ischemia occluded for different durations
}

\author{
WENTING DING ${ }^{*}$, LEQUAN ZHOU*, WEI LIU, LI GUAN, XIAOYING LI, HAIMEI LIU, \\ FUMAN YAN, JINWEN XU, WEIYONG ZENG and MIN QIU
}

Department of Physiology, College of Fundamental Medical Science, Guangzhou University of Chinese Medicine, Guangzhou, Guangdong 510006, P.R. China

Received July 11, 2013; Accepted March 7, 2014

DOI: $10.3892 / \mathrm{mmr} .2014 .2075$

\begin{abstract}
Protectants and executioners have been demonstrated to be used by gap junctions in focal cerebral ischemia. Certain researchers hypothesized that the opposite role of gap junctions may be associated with the injury extent, which has been demonstrated to be highly correlated with occlusion duration. In order to examine this hypothesis directly, the effects of octanol, a frequently used drug, were examined to investigate the role of gap junctions, in rats following middle cerebral artery occlusion (MCAO) for $30 \mathrm{~min} / 2 \mathrm{~h}$ and $24 \mathrm{~h}$ reperfusion, respectively. Octanol significantly reduced the infarct volume following $2 \mathrm{~h}$ of occlusion concomitant with lower neurological deficits, whereas it enlarged the infarct volume following $30 \mathrm{~min}$ of occlusion. Consistently, octanol attenuated the number of transferase dUTP nick-end labeling (TUNEL) positive neurons in the hippocampal CA1 region following $2 \mathrm{~h}$ of occlusion, while opposite effects were observed for $30 \mathrm{~min}$ of occlusion. Further immunohistochemical studies demonstrated that the expression of B-cell leukemia-2 (Bcl-2, anti-apoptotic protein) was upregulated and that Bcl-2-associated $\mathrm{X}$ (Bax, proapoptotic protein) was downregulated following $2 \mathrm{~h}$ of occlusion in the octanol group compared with the ischemic group. Conversely, octanol downregulated the expression of the $\mathrm{Bcl}-2$ protein concomitant with increased Bax protein following $30 \mathrm{~min}$ of occlusion. These
\end{abstract}

Correspondence to: Dr Wei Liu, Department of Physiology, College of Fundamental Medical Science, Guangzhou University of Chinese Medicine, 232 Waihuan East Road, Guangzhou, Guangdong 510006, P.R. China

E-mail: weiliu1980@yahoo.com

*Contributed equally

Abbreviations: MCAO, middle cerebral artery occlusion; OGD, oxygen-glucose deprivation; TUNEL, transferase dUTP nick-end labeling; IR, immunoreactive; Bcl-2, B-cell leukemia-2; Bax, Bcl-2associated $\mathrm{X}$

Key words: gap junctions, focal cerebral ischemia, octanol, occlusion time, astrocyte results indicated that the gap junction blocker octanol can protect against ischemic injury following long-term occlusion, however, can aggravate ischemic injury following short-term occlusion.

\section{Introduction}

Gap junctions connect the interior of cells and are important in the maintenance of tissue homeostasis. Certain deleterious metabolites involved in cerebral ischemia and apoptotic associated molecules, including $\mathrm{Ca}^{2+}, \mathrm{IP}_{3}$, ATP and cAMP, may be small enough to pass through gap junctions and modulate cell death of the neighboring cells, enhancing the spreading of injury $(1,2)$. Alternatively, the network of gap junctions could prevent the death of injured cells by the buffering of toxic metabolites to healthy surrounding cells (3). Thus, gap junctions could have damaging and protective effects (4).

There is increasing evidence that suppressing gap junctions is able to reduce the spread of damage following ischemia (5). For example, the gap junction blocker octanol restricted the flow of undesirable neurotoxins and significantly decreased the spread of cell death (6). Similarly, connexin 43 (the predominant connexin in gap junctions) heterozygous mice demonstrated reduced shrinkage and metabolite abnormality in the ipsilateral hippocampus following middle cerebral artery occlusion (MCAO) (7). Lin et al demonstrated in vitro that the resistance of $\mathrm{C} 6$ cells to calcium overload and oxidative stress was compromised when they formed gap junctions with more vulnerable cells (8). Despite the detrimental role of gap junctions, it is noteworthy that gap junctions may mediate survival or be protective for adjacent cells. In mixed astrocyte neuron cultures, the inhibition of astrocyte coupling with gap junction blockers increased neuronal vulnerability to oxidative stress or glutamic acid toxicity $(9,10)$. Consistently, connexin 43 overexpression substantially suppressed zinc (released during ischemia) toxicity whereas its knockdown caused a significant enhancement of the toxicity (11).

Certain investigators proposed that the opposite role of gap junctions may be associated with the extent of neuronal death (12), which was highly correlated with the duration of ischemia $(13,14)$. Therefore, the present study examined whether the opposite role of gap junctions was associated with occlusion time. In order to examine this issue, a gap junction 
blocker octanol was used, which has been administered to investigate the role of gap junctions in numerous studies $(6,15)$. The effects of octanol in MCAO was evaluated using neurological deficits, infarct volume and transferase dUTP nick-end labeling (TUNEL) staining. To further investigate the mechanism of the opposite roles of octanol, the expression of Bcl-2 and Bax was studied, which play opposite roles in apoptosis (16). The present study provides evidence that may be used to further elucidate the opposite role of gap junctions in ischemia.

\section{Materials and methods}

Animal model of cerebral ischemia. Male adult Sprague-Dawley rats weighing 250-280 g were maintained in a temperature- and light-controlled environment with a $12 \mathrm{~h}$ light/dark cycle. All experiments were performed in accordance with the NIH guidelines and approval by the Animal Care and Use Committee of the Guangzhou University of Chinese Medicine (Guangzhou, Guangdong, China) and all efforts were made to minimize animal suffering.

A focal cerebral ischemic rat model was induced by MCAO as previously described (17). In brief, the rats were anesthetized with $350 \mathrm{mg} / \mathrm{kg}$ of chloral hydrate via intraperitoneal injection. The right carotid bifurcation was exposed and the external carotid artery was coagulated distal to the bifurcation. A 4-0 nylon monofilament suture with a rounded tip was introduced into the internal carotid artery through the stump of the external carotid artery and gently advanced for $20 \mathrm{~mm}$. Following $30 \mathrm{~min} / 2 \mathrm{~h}$ of occlusion, the filament was gently withdrawn and the incision was closed (18). During the whole process, the rat rectal temperature was maintained at $37^{\circ} \mathrm{C}$ by placing the animals on a heating bed. In the sham-operated rats, the common carotid was exposed without ligation.

Drug treatment. Octanol purchased from Sigma (St. Louis, MO, USA) was dissolved in dimethyl sulfoxide (DMSO; $0.005 \% \mathrm{v} / \mathrm{v}$ ) and a final concentration of $5 \mathrm{mmol} / \mathrm{kg}$ was administered intraperitoneally $30 \mathrm{~min}$ prior to ischemia (6). The vehicle-treated rats were administered equal volumes of DMSO.

Measurement of neurological deficits. An independent observer performed the assessment of the neurological deficit score: 0 , no neurological deficit; 1 , failure to fully extend left forepaw; 2, circling to the left; 3 , falling to the left; 4 , loss of spontaneous walking with a depressed level of consciousness and 5, dead (17).

Measurement of infarct volume. Following reperfusion for $24 \mathrm{~h}$, rats were overdosed with sodium pentobarbital $(100 \mathrm{mg} / \mathrm{kg})$. The brains were quickly removed and cut into coronal slices of $2 \mathrm{~mm}$ in thickness. The sections were immersed in a $2 \%$ solution of 2,3,5-triphenyltetrazolium chloride (TTC; Mym Biological Technology, Andhra Pradesh, India) for $20 \mathrm{~min}$ at $37^{\circ} \mathrm{C}$ and then fixed with $4 \%$ paraformaldehyde. The infarct area was measured by each slice using a computerized image analysis system (Image-Pro-Plus; Media Cybernetics, Silver Spring, MD, USA). Infarct areas of all sections were added to derive the total infarct area, which was multiplied by the thickness. The infarct volume was expressed as a percentage of the ipsilateral hemispheric volume (\%).

Tissue preparation and TUNEL assay. Rats were deeply anesthetized with sodium pentobarbital and perfused through the ascending aorta with cold saline, followed by $4 \%$ paraformaldehyde in 0.1 M of phosphate-buffered saline (PBS; $\mathrm{pH}$ 7.2-7.4, $4^{\circ} \mathrm{C}$ ). The brains were cytoprotected by 15 and $30 \%$ sucrose sequentially. Coronal sections were cut on a freezing microtome at a thickness of $15 \mu \mathrm{m}$ starting at $+1.60 \mathrm{~mm}$ to $-4.80 \mathrm{~mm}$ from the bregma and then were mounted onto polylysine-coated slides. Intervening sections were air dried, then stored at $-80^{\circ} \mathrm{C}$ until future use. TUNEL staining was processed using an in situ cell death detection kit (Roche Molecular Biochemicals, Mannheim, Germany) according to the manufacturer's instructions. Images were captured using an Olympus microscope (Olympus, Tokyo, Japan) and the number of TUNEL-positive neurons within the CA1 subfield was counted.

Immunohistochemistry. Brain sections (15 $\mu \mathrm{m}$ thickness) were mounted with $2 \%$ goat serum in $0.1 \mathrm{M}$ of Tris buffered saline (TBS) $/ 0.3 \%$ Triton $\mathrm{X}-100$ for $1 \mathrm{~h}$ at room temperature and incubated overnight at $4{ }^{\circ} \mathrm{C}$ with the primary antibody for Bcl-2/Bax (1:100; Santa Cruz Biotechnology, Inc., Santa Cruz, CA, USA). Following washing in TBS, sections were incubated with biotinylated goat anti-rabbit $\operatorname{IgG}$ and further processed with the ABC method (Vector, San Diego, CA, USA). The stained sections were captured with a CCD spot camera. Non-specific staining was determined by omitting the primary antibodies.

To quantify Bcl-2 and Bax immunoreactivity (IR), the measurement was performed with a computerized image analysis system (Image-Pro-Plus, Media Cybernetics) according to the previously described method (19). Briefly, a density threshold was set above the background level firstly to identify a positively stained structure and the area occupied by these structures was measured as positive area. An average percentage of Bcl-2-IR or Bax-IR area to the total outlined area was obtained. Five to six animals were included in each group for the quantification of immunohistochemistry.

Quantification and statistical analysis. Observers blinded to the experimental conditions, evaluated the outcome measures. All data are presented as the mean \pm SEM. The Student's t-test was used to compare the difference between the vehicle group and the octanol group. All analysis was performed using the Statistical Package for Social Sciences (SPSS, version 13.0 for Windows). $\mathrm{P}<0.05$ was considered to indicate a statistically significant difference.

\section{Results}

Neurological deficits. The effect of octanol was evaluated by the Zea Longa test. As shown in Fig. 1, octanol significantly lowered the neurological deficits compared with the vehicle group following $2 \mathrm{~h}$ of ischemia and $24 \mathrm{~h}$ of reperfusion $(\mathrm{P}=0.028)$. As for the $30 \mathrm{~min}$ ischemia group, although the mean of the neurological deficit scores was higher in the octanol group, the difference between the two groups was not statistically significant $(\mathrm{P}=0.181)$. 


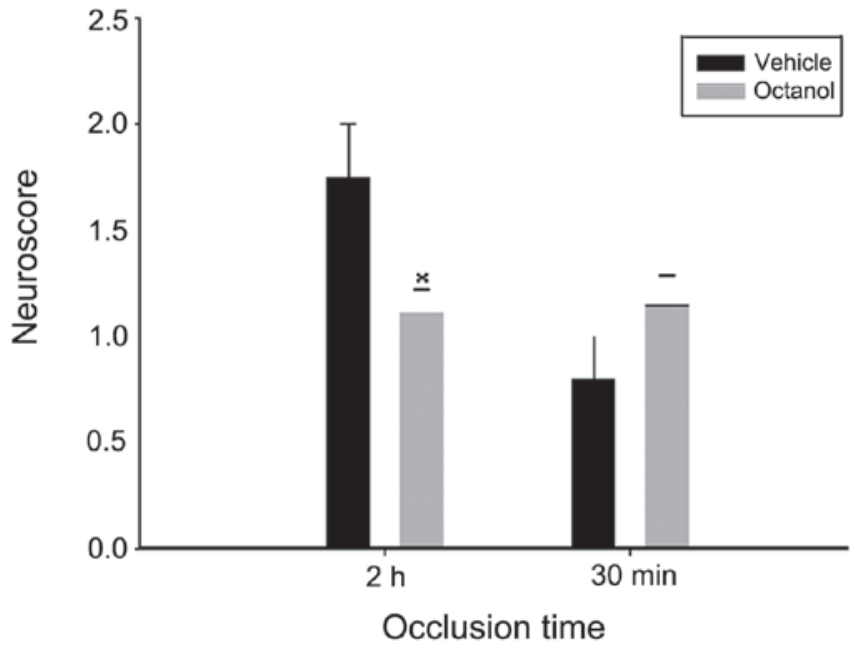

Figure 1. Evaluation of neurological impairment following treatment. Octanol pretreatment significantly decreased the neurological deficit score in ischemia for $2 \mathrm{~h}$. ${ }^{*} \mathrm{P}<0.05$ vs. the vehicle group for $2 \mathrm{~h}$.

Infarct volume evaluation. Representative images of TTC staining were shown in Fig. 2. The infarct volume following $2 \mathrm{~h}$ of occlusion was reduced by octanol pretreatment compared with the vehicle group $(\mathrm{P}=0.001)$. Conversely, a larger stroke volume was observed in the octanol group following $30 \mathrm{~min}$ of occlusion compared with that in the vehicle group $(\mathrm{P}=0.025)$.

TUNEL assay. A moderate number of markedly TUNEL-labeled CA1 pyramidal neurons were identified in the CA1 region in the vehicle group following ischemia for $2 \mathrm{~h}$ and reperfusion for $24 \mathrm{~h}$. However, the number of TUNELpositive neurons in the CA1 region of rats pretreated with octanol was markedly decreased ( $\mathrm{t}=7.812, \mathrm{P}<0.001$; Fig. 3). Notably, in the $30 \mathrm{~min}$ ischemia group, octanol significantly increased the number of TUNEL-positive cells compared with the vehicle group ( $\mathrm{t}=4.595, \mathrm{P}=0.002$; Fig. 3 ).

Expression of $\mathrm{Bcl}-2$ and Bax. Apoptosis contributes to delayed neuronal death causing expansion of the stroke lesion, therefore we investigated the expression of anti-apoptotic $\mathrm{Bcl}-2$ and pro-apoptotic Bax expression. In the $2 \mathrm{~h}$ ischemia group, compared with the vehicle group, octanol significantly increased the expression of $\mathrm{Bcl}-2(\mathrm{t}=6.151, \mathrm{P}<0.001)$, however decreased the expression of $\operatorname{Bax}(\mathrm{t}=2.768, \mathrm{P}=0.016$; Figs. 4 and 5). However, in the $30 \mathrm{~min}$ ischemia group, the rats treated with octanol demonstrated that, accompanied by a decrease in $\mathrm{Bcl}-2$ expression ( $\mathrm{t}=2.907, \mathrm{P}=0.012)$, Bax expression was markedly increased $(\mathrm{t}=4.149, \mathrm{P}=0.001$; Figs. 4 and 5).

\section{Discussion}

The present study demonstrated and compared for the first time, to the best of our knowledge, the effect of the gap junction blocker octanol in MCAO for $2 \mathrm{~h}$ and $30 \mathrm{~min}$, respectively. Compared with the vehicle-treated rats, octanol attenuated the ischemia injury induced by $2 \mathrm{~h}$ of occlusion demonstrated by the neurological deficit, infarct volume and
TUNEL staining, whereas it aggravated the ischemia injury induced by $30 \mathrm{~min}$ of occlusion, indicating that octanol was protective following $2 \mathrm{~h}$ of occlusion but harmful following $30 \mathrm{~min}$ of occlusion. Octanol, an eight-carbon aliphatic alcohol, is a relatively specific inhibitor of gap junction permeability and is frequently used to study the role of gap junctions. Although certain studies have demonstrated that octanol can interfere with synaptic transmission (20), it is hard to interpret these two opposite effects with gap junction-independent mechanisms. The opposite role of octanol appears to be closely associated with the function of gap junctions (21). For example, gap junctions could be neuroprotective since adjacent healthy cells may act as an effective spatial buffer against the extracellular accumulation of neurotoxic substances $(22,23)$. By contrast, they could also be harmful when neighboring cells are unable to aid in the clearance of the neurotoxic substances, which could depolarize a large number of neurons/glia promoting the release of glutamate and therefore causing aggravated injury (24).

Gap junctions have been revealed to be protective in the model of hypoxic preconditioning (subthreshold insults) (25), MCAO above and below the rhinal fissure (26-29) and MCAO for 30 min (30). By contrast, previous investigations also demonstrated that gap junctions were harmful in the model of four vessels occlusion (31), clamping the common carotid arteries and lowering the mean arterial blood pressure to $40 \mathrm{mmHg}$ (6), MCAO for $16 \mathrm{~h}$ (32) and oxygen-glucose deprivation (OGD) for 6 to 10 days (33). As the intensity and/or the duration of the ischemic episode increases, the predominant function of gap junctions may be different. For example, when the buffering effect is stronger than the harmful factors' propagation in the condition that injury is mild, gap junctions may be neuroprotective; otherwise gap junctions may be harmful. In the present study, the infarct volume following $30 \mathrm{~min}$ of occlusion was only $6.4 \%$, thus gap junctions were efficient in buffering harmful factors. By contrast, $2 \mathrm{~h}$ of occlusion induced a much larger infarct volume, therefore gap junctions could not buffer harmful factors efficiently and more neurotoxic substances propagated to neighbouring cells aggravating the injury instead.

Notably, while octanol significantly increased the infarct volume and cell death following $30 \mathrm{~min}$ of occlusion, no statistical differences in neurological deficits were identified between the octanol group and the vehicle group. Similarly, Longa et al demonstrated that the infarct area following $2 \mathrm{~h}$ of temporary MCAO were $15.7 \%$ smaller than that following permanent MCAO, however the neurological deficit was not significantly reduced (17). In light of these findings, the neurological score was not closely associated with the infarct volume as occasionally the changes in infarct volume may not be enough to create differences in the neurological test.

To explore the possible mechanisms, we detected Bcl-2 and Bax immunoreactivity, respectively, which are two opposite apoptotic factors. Bcl-2 can help neuronal survival and protect brain tissue from ischemic injury $(34,35)$. It acted upstream to prevent the activation of caspases, inhibited free radical formation and regulated calcium sequestration (36). However, Bax inhibition reduced apoptotic neuronal injury in the hippocampal CA1 region and behavioral deficits following global ischemia $(37,38)$. In the present study, we 

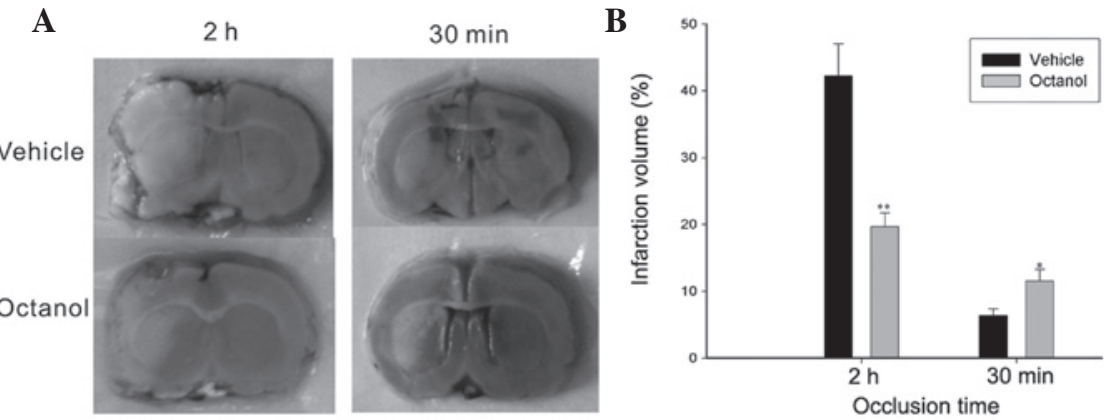

Figure 2. Effect of octanol on infarct volume following ischemia for $2 \mathrm{~h} / 30 \mathrm{~min}$ and $24 \mathrm{~h}$ reperfusion. (A) Infarct volumes of the octanol- and vehicle-treated groups. (B) Changes between the vehicle group and the octanol group were compared using the percentage of infarct area of the ipsilateral hemisphere. ${ }^{* *} \mathrm{P}<0.01$, vs. the vehicle group for $2 \mathrm{~h} ;{ }^{*} \mathrm{P}<0.05$ vs. the vehicle group for $30 \mathrm{~min}$.
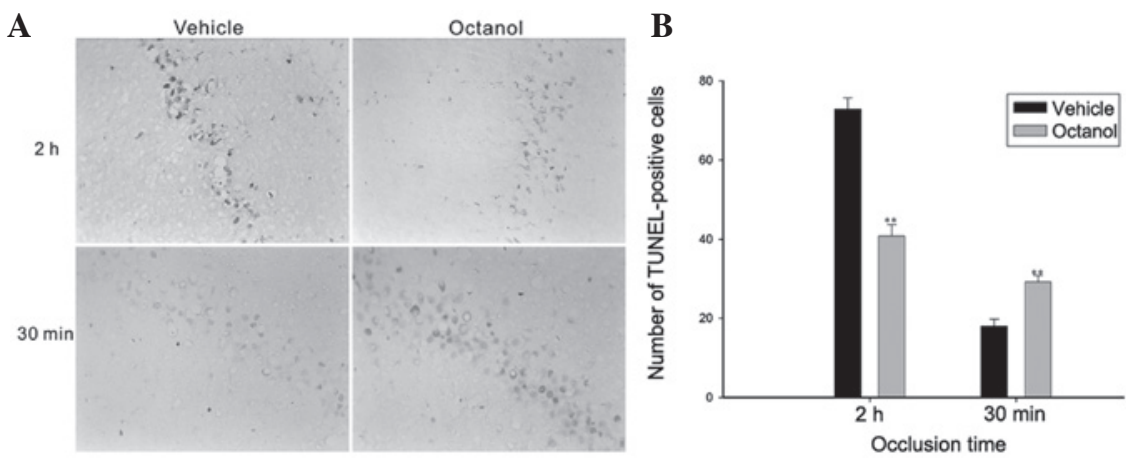

Figure 3. Effect of octanol on DNA fragmentation in CA1 neurons. (A) In the $2 \mathrm{~h}$ ischemia group, octanol decreased TUNEL- positive cells; however, in the $30 \mathrm{~min}$ ischemia group, octanol increased TUNEL- positive cells (magnification, $\mathrm{x} 400$ ). (B) Statistical results are shown. ${ }^{* *} \mathrm{P}<0.01$, respectively vs. the vehicle group. TUNEL, transferase dUTP nick-end labeling.
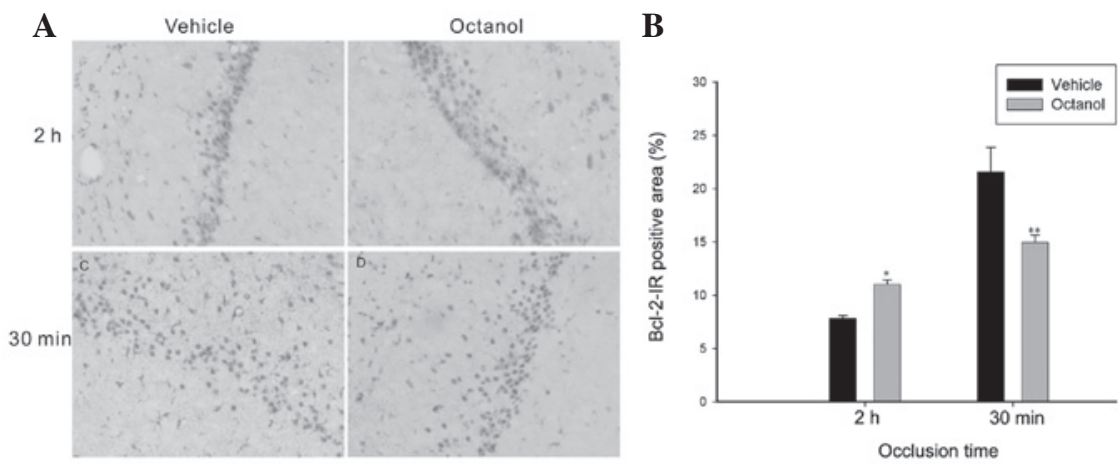

Figure 4. Expression of anti-apoptotic Bcl-2 in different groups. (A) Expression of Bcl-2 in the CA1 area following ischemia for $2 \mathrm{~h} / 30 \mathrm{~min}$ and $24 \mathrm{~h}$ reperfusion (magnification, $\mathrm{x} 400$ ). (B) There were significant differences between the vehicle group and the octanol group, respectively. " $\mathrm{P}<0.05$, vs. the vehicle group for $2 \mathrm{~h} ;{ }^{* *} \mathrm{P}<0.01$ vs. the vehicle group for $30 \mathrm{~min}$. Bcl-2, B-cell leukemia-2.

A

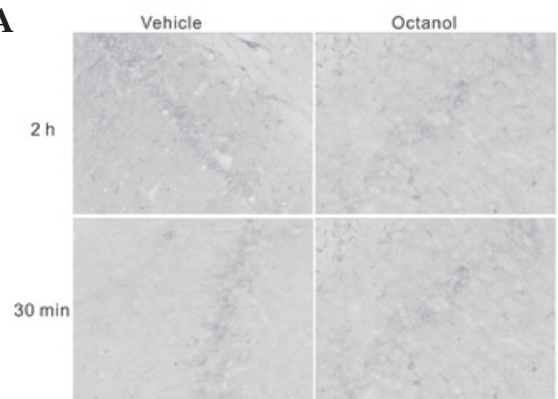

B

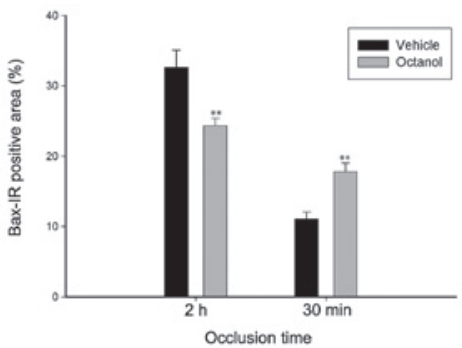

Figure 5. Expression of pro-apoptotic Bax in different groups. (A) Expression of Bax in the CA1 area following ischemia for $2 \mathrm{~h} / 30 \mathrm{~min}$ and $24 \mathrm{~h}$ reperfusion (magnification, $\mathrm{x} 400$ ). (B) There were significant differences between the vehicle group and the octanol group, respectively. ${ }^{* *} \mathrm{P}<0.01$ respectively vs. the vehicle group. Bax, Bcl-2-associated X. 
revealed that octanol induced $\mathrm{Bcl}-2$ expression and attenuated Bax expression in MCAO for $2 \mathrm{~h}$. By contrast, it inhibited Bcl-2 expression and increased Bax staining in MCAO for $30 \mathrm{~min}$. These results demonstrated that the protective role of octanol in ischemia for $2 \mathrm{~h}$ may be associated with its ability to prevent apoptosis via facilitation of the anti-apoptotic factor Bcl-2 or inhibition of the apoptotic biochemical factor Bax. Conversly, the decreased Bcl-2 and increased Bax contributed to apoptosis, thus resulting in a harmful role of octanol in ischemia for $30 \mathrm{~min}$. We are not completely certain that the opposite roles of octanol on Bcl-2 and Bax is directly mediated by gap junction activity. However, the consistency between ischemia injury and the corresponding $\mathrm{Bcl}-2 / \mathrm{Bax}$ expression in ischemia for different durations, to a certain extent, verifies that the present results are mainly attributed to the effect of octanol on gap junctions.

In summary, the present study investigated and compared the effects of octanol in MCAO for the first time, to the best of our knowledge. Our results demonstrated that the gap junction blocker octanol played opposite roles in MCAO for different durations, which was demonstrated by the neurological outcome, infarct size and TUNEL staining. In addition, the function of octanol was at least in part mediated through the counter-regulation of Bcl-2 and Bax. These data provide a novel perspective for the role of gap junctions in cerebral ischemia and may be beneficial for the potential clinical treatment of various ischemic conditions. However, more studies are required to examine this, including using other blockers of gap junctions or connexin 43 deficient mice.

\section{Acknowledgements}

This study was supported by the National Nature Science Foundation (no. 81202817), the Guangdong Province Medical Research Foundation (no. B2011123), the Natural Science Foundation of Guangdong Province (no. S2012040007519) and the Guangdong College of outstanding youth innovation talent training project (no. LYM11042; no. 2012LYM0045).

\section{References}

1. Krysko DV, Leybaert L, Vandenabeele P and D'Herde K: Gap junctions and the propagation of cell survival and cell death signals. Apoptosis 10: 459-469, 2005.

2. Peixoto PM, Ryu SY, Pruzansky DP, Kuriakose M, Gilmore A and Kinnally KW: Mitochondrial apoptosis is amplified through gap junctions. Biochem Biophys Res Commun 390 $38-43,2009$.

3. Sahores M and Mendoza-Naranjo A: Gap junctions as therapeutic targets in brain injury following hypoxia-ischemia. Recent Pat CNS Drug Discov 3: 209-315, 2008.

4. Spray DC, Hanstein R, Lopez-Quintero SV, Stout RF Jr, Suadicani SO and Thi MM: Gap junctions and Bystander effects: Good Samaritans and executioners. Wiley Interdiscip Rev Membr Transp Signal 2: 1-15, 2013.

5. Davidson JO, Green CR, Bennel L, Nicholson LF, Danesh-Meyer H, O'Carroll SJ and Gunn AJ: A key role for connexin hemichannels in spreading ischemic brain injury. Curr Drug Targets 14: 36-46, 2013.

6. Rami A, Volkmann T and Winckler J: Effective reduction of neuronal death by inhibiting gap junctional intercellular communication in a rodent model of global transient cerebral ischemia. Exp Neurol 170: 297-304, 2001.

7. Xie M, Yi C, Luo X, et al: Glial gap junctionsal communication involvement in hippocampal damage after middle cerebral artery occlusion. Ann Neurol 70: 121-132, 2011.
8. Lin JH, Weigel H, Cotrina ML, et al: Gap-junction-mediated propagation and amplification of cell injury. Nat Neurosci 1: 494-500, 1998

9. Blanc EM, Bruce-Keller AJ and Mattson MP: Astrocytic gap junctional communication decreases neuronal vulnerability to oxidative stress-induced disruption of $\mathrm{Ca}^{2+}$ homeostasis and cell death. J Neurochem 70: 958-970, 1998.

10. Ozog MA, Siushansian R and Naus CC: Blocking gap junctional coupling increases glutamate-induced neurotoxicity in neuron-astrocyte co-cultures. J Neuropathol Exp Neurol 61: 132-141, 2002.

11. Lee J, Yim YS, Ko SJ, Kim DG and Kim CH: Gap junctions contribute to astrocytic resistance against zinc toxicity. Brain Res Bull 86: 314-318, 2011.

12. Chew SS, Johnson CS, Green CR and Danesh-Meyer HV: Role of connexin43 in central nervous system injury. Exp Neurol 225: 250-261, 2010.

13. Bendel O, Alkass K, Bueters T, von Euler M and von Euler G: Reproducible loss of CA1 neurons following carotid artery occlusion combined with halothane-induced hypotension. Brain Res 1033: 135-142, 2005.

14. Colbourne F, Li H and Buchan AM: Continuing postischemic neuronal death in CA1: influence of ischemia duration and cytoprotective doses of NBQX and SNX-111 in rats. Stroke 30: 662-668, 1999 .

15. Rawanduzy A, Hansen A, Hansen TW and Nedergaard M: Effective reduction of infarct volume by gap junction blockade in a rodent model of stroke. J Neurosurg 87: 916-920, 1997.

16. Kaido T, Kempski O, Heimann A, Heers C and Bartsch D: Cluster analysis of mRNA expression levels identifies multiple sequential patterns following focal cerebral ischemia. Turk Neurosurg 22: 441-447, 2012.

17. Longa EZ, Weinstein PR, Carlson S and Cummins R: Reversible middle cerebral artery occlusion without craniectomy in rats. Stroke 20: 84-91, 1989.

18. Zivin JA: Factors determining the therapeutic window for stroke. Neurology 50: 599-603, 1998

19. George A, Marziniak M, Schäfers M, Toyka KV and Sommer C: Thalidomide treatment in chronic constrictive neuropathy decreases endoneurial tumor necrosis factor-alpha, increases interleukin-10 and has long-term effects on spinal cord dorsal horn met-enkephalin. Pain 88: 267-275, 2000.

20. Narahashi T, Aistrup GL, Lindstrom JM, Marszalec W, Nagata K, Wang F and Yeh JZ: Ion channel modulation as the basis for general anesthesia. Toxicol Lett 100: 185-191, 1998.

21. Contreras JE, Sánchez HA, Véliz LP, Bukauskas FF, Bennett MV and Sáez JC: Role of connexin-based gap junction channels and hemichannels in ischemia-induced cell death in nervous tissue.Brain Res Brain Res Rev 47: 290-303, 2004.

22. Retamal MA, Schalper KA, Shoji KF, Orellana JA, Bennett MV and Sáez JC: Possible involvement of different connexin43 domains in plasma membrane permeabilization induced by ischemia-reperfusion. J Membr Biol 218: 49-63, 2007.

23. Rouach N, Avignone E, Même W, Koulakoff A, Venance L, Blomstrand F and Giaume C: Gap junctions and connexin expression in the normal and pathological central nervous system. Biol Cell 94: 457-475, 2002.

24. Talhouk RS, Zeinieh MP, Mikati MA and El-Sabban ME: Gap junctional intercellular communication in hypoxia-ischemia-induced neuronal injury. Prog Neurobiol 84: 57-76, 2008

25. Lin JH, Lou N, Kang N, et al: A central role of connexin 43 in hypoxic preconditioning. J Neurosci 28: 681-695, 2008.

26. Nakase T, Fushiki S and Naus CC: Astrocytic gap junctions composed of connexin 43 reduce apoptotic neuronal damage in cerebral ischemia. Stroke 34: 1987-1993, 2003.

27. Nakase T, Fushiki S, Söhl G, Theis M, Willecke K and Naus CC: Neuroprotective role of astrocytic gap junctions in ischemic stroke. Cell Commun Adhes 10: 413-417, 2003.

28. Nakase T, Sähl G, Theis M, Willecke K and Naus CC: Increased apoptosis and inflammation after focal brain ischemia in mice lacking connexin43 in astrocytes. Am J Pathol 164: 2067-2075, 2004.

29. Siushansian R, Bechberger JF, Cechetto DF, Hachinski VC and Naus CC: Connexin43 null mutation increases infarct size after stroke. J Comp Neurol 440: 387-394, 2001.

30. Wang F, Hai J and Jing Y: Gap junctions communication involved in brain protection following focal ischemia and reperfusion in rats. Neural Regen Res 9: 677-682, 2009 (In Chinese). 
31. Perez Velazquez JL, Kokarovtseva L, Sarbaziha R, Jeyapalan $\mathrm{Z}$ and Leshchenko Y: Role of gap junctionsal coupling in astrocytic networks in the determination of global ischaemia-induced oxidative stress and hippocampal damage. Eur J Neurosci 23: 1-10, 2006.

32. Saito R, Graf R, Hübel K, Fujita T, Rosner G and Heiss WD: Reduction of infarct volume by halothane: effect on cerebral blood flow or perifocal spreading depression-like depolarizations. J Cereb Blood Flow Metab 17: 857-864, 1997.

33. de Pina-Benabou MH, Szostak V, Kyrozis A, et al: Blockade of gap junctions in vivo provides neuroprotection after perinatal global ischemia. Stroke 36: 2232-2237, 2005.

34. Martinou JC, Dubois-Dauphin M, Staple JK, et al: Overexpression of BCL-2 in transgenic mice protects neurons from naturally occurring cell death and experimental ischemia. Neuron 13: 1017-1030, 1994.
35. Chen J, Simon RP, Nagayama T, Zhu R, Loeffert JE, Watkins SC and Graham SH: Suppression of endogenous bcl-2 expression by antisense treatment exacerbates ischemic neuronal death. J Cereb Blood Flow Metab 20: 1033-1039, 2000.

36. MacManus JP and Linnik MD: Gene expression induced by cerebral ischemia: an apoptotic perspective. J Cereb Blood Flow Metab 17: 815-832, 1997.

37. Han B, Wang Q, Cui G, Shen X and Zhu Z: Post-treatment of Bax-inhibiting peptide reduces neuronal death and behavioral deficits following global cerebral ischemia. Neurochem Int 58: 224-233, 2011.

38. Xi HJ, Zhang TH, Tao T, Song CY, Lu SJ, Cui XG and Yue ZY: Propofol improved neurobehavioral outcome of cerebral ischemia-reperfusion rats by regulating Bcl-2 and Bax expression. Brain Res 1410: 24-32, 2011. 